Conclusions: As the population ages, the burden of chronic disease in the population increases. During a disaster with large numbers of displaced persons, accommodations for such persons must be accounted for in order to prevent a second disaster related to de-compensation of those with chronic health problems in the ACS shelter. Understanding the population needs beforehand can mitigate the effects of displacement on this population.

Prehosp Disaster Med 2011;26(Suppl. 1):s39-s40

doi:10.1017/S1049023X11001403

(A140) An Analysis of National Survey on Disaster Drill by Emergency Medical Centers in Korea

S.J. Wang

Emergency Medicine, Seoul, Korea

Introduction: Disaster drills have been performed officially for disaster medical preparedness by wide regional emergency medical centers in Korea. Government evaluates these disaster drills every year, but this evaluation is performed based on administrative aspect not disaster medical aspect. Also there are insufficient number of research on disaster drill not on disaster itself, especially national level. So these disaster medical drills were analyzed and reevaluated.

Methods: Disaster drills performed by wide regional emergency medical centers in Korea were evaluated and analyzed from August 2006 to July 2008. Disaster drill planning, performance, kinds of disaster drill, contents of drill and resources of drill were collected and analyzed in medical aspect based on the disaster drill report and additional compensation of incomplete data by direct contact with the participating institutions. The appropriateness and evaluation results referred to the guideline of National Emergency Medical Center of Korea.

Results: All the wild regional emergency medical centers had their own disaster drill planning and resource application planning for drills and real disasters. Most of disaster drill planning were appropriate, but disaster facility was the weakest point in the planning. Types of disasters in disaster drills were fire and structural collapse(44.4\%), special disaster(13.7\%), natural disaster(1.7\%). Average duration of disaster drills were 6.12 hours and 1.26 days. Real field drill rate was $80.3 \%$. Sixty five percent of drills were connected to multiple institutions or organizations other than hospitals. Number of participating persons from wide regional emergency medical centers were 21 on average.

Conclusions: Medical situation on disaster drills is that there are various levels and kinds of disaster drills done by wide regional emergency medical centers, so the quality and quantity should be enforced in low level centers. It is recommended that international situation or database can be extracted based on this research.

Prehosp Disaster Med 2011;26(Suppl. 1):s40

doi:10.1017/S1049023X11001415

\section{(A141) Hospital Preparedness for a Large Scale Biological} Drill

O. Benin-Goren, ${ }^{1}$ E. Miller, ${ }^{2}$ I. Dalla ${ }^{2}$

1. Nursing Division Management, Tel Aviv, Israel

2. Disaster Preparedness Team, Tel Aviv, Israel

Background: During the past four years large scale biological exercises took place in several districts of the state of
Israel. The drills included hospitals, Health Maintenance Organizations (HMOs), Emergency Medical Services (EMS), public health district offices and interface agencies such as Israel Defense Forces (IDF) Home Front Command (HFC).

Discussion and Observations: On January 2010 a comprehensive biological exercise was conducted in Tel Aviv. Tel Aviv Sourasky Medical Center (TASMC) together with the agencies mentioned above practiced the hospital competing with Exceptional Biological Event. New elements, which had never been inspected before, were evaluated in this drill:

- Activating of a triage point at the hospital gate.

- Opening a special registration point.

- Staff protection from biological agents.

- Activating a separate Emergency Department (ED) for bio- threat.

- Detection and Containment ward.

- Protected elevators and passageways

- Interface agencies in and out the hospital.

Designated physicians, nurses and paramedical team, that practiced other events in the past, needed to be trained and practiced in order to be ready for a biological event. Wards that usually did not participate in such drills, needed to take part in this particular drill, while the routine work continued. This paper presents the hospital preparation for the drill, the methodology of training and preparedness, as well as the outcome of the drill. Prehosp Disaster Med 2011;26(Suppl. 1):s40 doi:10.1017/S1049023X11001427

(A142) Simulated Evacuation of Three Critical Hospital Departments: A Comparison

E.L. Dhondt, ${ }^{1}$ D. Lauwaert, ${ }^{1}$ C. Hendrick ${ }^{2}$

1. Department of Emergency and Disaster Medicine, Brussels, Belgium

2. Leuven, Belgium

Background: According to the Belgian Hospital Disaster Planning Act, all hospitals are required to have written disaster plans and to routinely conduct annual disaster drills. In 2010, three neighboring hospitals organized independently from each other an evacuation exercise of a critical care department (CCD): two university hospitals of a Dialysis Center and a One-day Surgery Clinic respectively and the military hospital of a Burn Unit.

Aim: To compare these CCD's evacuation plans and drills and the overall hospital emergency incident response and command system.

Methods and Results: Conducting an evacuation exercise in a CCD, moving vulnerable highly dependent people towards an alternative shelter site is challenging, causing an important burden to ongoing medical specialist care, working staff and critical infrastructure. In all three CCD, it was decided to conduct a simulated evacuation exercise following an internal fire, thereby deploying fashioned simulated patients and visitors but bringing into action the regular attending medical, nursing and logistic staff. In each hospital a multidisciplinary design team was launched, consisting of the hospitals disaster preparedness coordinator, the EMS-staff, external emergency incident management and operational engineering experts. The appointed objectives for evaluation were the knowledge of the regular 
evacuation drills, especially the clearance of an intensive care or an operating room; access to evacuation routes; visibility of safety guidelines; need of specific evacuation equipment for the movement of patients; mission and tasks of the hospital's first response team and the medical incident manager; communication and information flow and the establishment of the hospital's coordination committee.

Conclusion: 1. Simulated hospital evacuation exercises increased the hospital emergency preparedness, awareness and response to disasters within the hospital, in particular in a CCD, otherwise difficult to assess. 2. All three CCD experienced the same challenges and identified similar flaws. 3. A hospital disaster exercise manual might be of valuable help. Prehosp Disaster Med 2011;26(Suppl. 1):s40-s41 doi:10.1017/S1049023X11001439

(A143) European Project SICMA (Simulation of Crisis Management Activities) for Medical Management of Maxi Emergency Trauma Patients S. Magalini, ${ }^{1}$ M. Di Mugno, ${ }^{1}$ A. De Gaetano, ${ }^{2}$

G. La Posta, ${ }^{2}$ D. Sermoneta, ${ }^{1}$ D. Gui ${ }^{1}$

1. Surgery, Rome, Italy

2. Rome, Italy

Introduction: Modern emergencies and disasters are progressively changing from relatively simple, predictable events controllable with standard management solutions to complex critical situations for which managers and first responders require innovative and affordable tools.

Methods: The European Project SICMA (Simulation of Crisis Management Activities) provides a modeling of the behavior of the entire Health Service System during field emergency operations, as well as the rules it operates by. Use of simulation technologies offers a significant improvement on current management activities allowing decision makers to confront several organizational alternatives not only with static situations but with evolving scenarios.

Results: SICMA simulates main structural and behavioral elements of maxi emergency and mass casualties, from individual casualties and evolution of their health status, activity of the police force and fire brigade on the accident site, crowd dynamics, sanitary personnel expertise, to ambulance and helicopter transportation depending on traffic and weather conditions. The system also simulates rescue doctrines (i.e. "Casualty Clearing Station" or "Scoop and run"), transportation priorities according to color codes, doctrines for assignment of new casualty to neighboring hospitals, hospital resources and involvement, final clinical outcome of individual casualties. Patient health status and physiological reserve of single casualty is based on the ABCD ATLS system, considering with a simple algorithm both level of damage and rate of worsening in time.

Conclusion: Utilizing this simulation system, managers who predispose organizational and logistic procedures may modify the main elements in order to identify the optimal resource allocation and the best procedures to save the most human lives.

Prehosp Disaster Med 2011;26(Suppl. 1):s41

doi:10.1017/S1049023X11001440
(A144) Health Status Casualty Model for Simulation of Crisis Management Activities (EU SICMA Project)

M. Di Mugno, ${ }^{1}$ S. Magalini, ${ }^{1}$ A. De Gaetano, ${ }^{2}$

G. La Posta ${ }^{2}$ D. Sermoneta, ${ }^{1}$ D. Gui ${ }^{1}$

1. Surgery, Rome, Italy

2. Rome, Italy

Introduction: The European Project SICMA (Simulation of Crisis Management Activities) provides a modeling of the behavior of the entire Health Service System during field emergency operations, as well as the rules it operates by. The first step toward chain procedure modelling in the management of major emergencies is the representation of a traumatized patient whose health status can be followed in time during simulation. Since management of the trauma patient follows criteria of stabilization of main physiological functions, a trauma patient model was developed based on fundamental pathophysiological functions independently of specific lesion characterization. Methods: Each patient's health status was modelled according to 5 parameters (ATLS): $\mathrm{A}$ (airway), $\mathrm{B}$ (breathing), $\mathrm{C}$ (circulatory), $\mathrm{D}$ (disability), E(Exposure). Patient samples are extracted from a 10.000 .000 patient database, generated by considering real anatomical lesions compatible with type and severity of considered scenarios (explosion, building collapse, fire, gunfight). Simulated lesion characteristics were then converted to pathophysiological parameters. Each physiological compensation parameter was represented by: (1) baseline value expressed as percentage of altered function; (2) function reduction rate over time, obtained by a mathematical approximation of clinical worsening. From level of function, rate of worsening and function-specific death thresholds, estimated time-to-death according to sustained damage is computed.

Results: This model allows simulation of evolution of patient health status both in absence of medical care, but also under therapy, in terms of immediate increment of each single parameter ("temporary" treatment), and of reduction or zeroing of parameter dec14rement rate ("definitive" treatment).

Conclusion: This model, based on evaluation of physiological parameters, presents an advantage over the consideration of single lesions, because simulating logical procedures that guide treatment choice in real situations can provide a more accurate assessment of casualities for those actors assigned to management of major emergencies.

Prehosp Disaster Med 2011;26(Suppl. 1):s41

doi:10.1017/S1049023X11001452

(A145) Simulation for the Assessment and Optimization of Medical Disaster Management

E.L. Dhondt, ${ }^{1}$ F. Van utterbeek, ${ }^{2}$ C. Ullrich, ${ }^{2}$

M. Debacker ${ }^{3}$

1. Emergency and Disaster Medicine, Brussels, Belgium

2. Brussels, Belgium

3. Military Hospital, Brussels, Belgium

Background: The ultimate goal of medical disaster management must be to predictably orchestrate transition from "standard of care" to "sufficiency of care" using evidence-based methods. However, neither descriptive reports of disaster responses nor epidemiological studies investigating disaster risk factors have been able to provide validated outcome measures as to what constitutes a "good" disaster response. Moreover, it either has 